\title{
LA GESTIÓN EN LOS PLANES INTEGRALES SISO EN EL SECTOR DE LA CONSTRUCCIÓN
}

\author{
SISO MANAGEMENT IN COMPREHENSIVE SCHEMES \\ IN THE CONSTRUCTION SECTOR
}

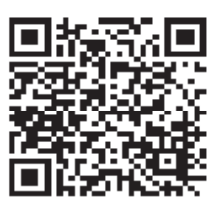

John Jairo Duque Arango ${ }^{1}$

1. Programa de Ingeniería Universidad del Quindío

Recibido: 2 Diciembre de 2015

Aceptado: 2 Marzo de 2016

*Correspondencia del autor: John Jairo Duque Arango. E-mail: jjduque@uniquindio.edu.co

\begin{abstract}
RESUMEN
El documento hace referencia al grado gestión de los planes SISO $^{1}$ por parte de la empresas del sector de la construcción, trabajo de investigación que permite determinar la aplicabilidad de los diversos programas que conforman el plan integral. La metodología utilizada como herramienta un software en java que de manera porcentual evalúa el grado de ejecución de cada una de los programas y sugiere los correctivos del caso. Los resultados obtenidos determinan el diagnóstico actual del sector y permiten el diseño de una guía de control que contiene una serie de actividades acordes al proceso constructivo. Las conclusiones abordan los aspectos de importancia del trabajo y propone una serie de recomendaciones a tener en cuenta en futuros proyectos para evitar situaciones y/o eventos de emergencia en la prevención de lesiones y accidentes del trabajo.
\end{abstract}

Palabras claves: Gestión, plan integral, programas, condiciones inseguras, diagnóstico, guía de control, lesiones, accidentes.

\begin{abstract}
The document refers to the degree SISO management plans by the companies in the construction sector, research that enables you to determine the applicability of the various programs that make up the comprehensive plan. The methodology used as tool software in java that percentage way evaluates the degree of implementation of each of the programs and suggests the remedies in the case. The results obtained determine the current diagnosis of the sector and enable the design of a control guide that contains a series of activities in accordance with the construction process. The conclusions dealt with aspects of importance of work and proposes a series of recommendations to take into account in future projects to avoid situations or events of emergency in the prevention of injuries and accidents at work.
\end{abstract}

Keywords: Management, comprehensive plan, programs, unsafe conditions, diagnosis, control, injuries, accidents Guide

\footnotetext{
${ }^{1 .}$ Documento resultado de la investigación: "La gestión de los planes siso en el sector de la construcción", presentado en la maestría en ingeniería con énfasis en gestión de la construcción, Universidad del Quindío convenio Universidad Eafit.
} 


\section{INTRODUCCION}

El objetivo de este documento es presentar los resultados obtenidos de la investigación acerca de la situación actual del sector de la construcción en seguridad industrial y salud ocupacional para los proyectos de construcción de vivienda en el Municipio de Armenia.

Este diagnóstico basa su desarrollo en datos estadísticos y cifras sobre accidentes de trabajo y enfermedades profesionales los cuales demuestran su alta incidencia y peligrosidad en el desempeño de la actividad, por lo tanto obliga a las empresas del sector a crear nuevos retos y estar preparados para enfrentarlos adaptándose al medio en que se realice el proyecto.

(Mincit, 2012), menciona que para este año el sector de la construcción ha tenido un crecimiento del $10.3 \%$, el cual se ha visto considerablemente afectado por niveles de accidentalidad significativos en Colombia con un 13\%, según (Fasecolda, 2010). Estos accidentes deben ser reportados al sistema general de riesgos en informes consolidados enviados por las diferentes compañías de seguros, con datos concretos sobre el tipo de lesión presente en las personas en el desempeño de diversas actividades.(ver Tabla $\mathrm{N}^{\circ} 1$ ).

Los datos estadísticos confirman que la accidentalidad laboral en comparativo del año 2010 - 2011 tiene un aumento del 3.5\% el cual corresponde a 36.365 eventos y en lo que respecta a casos de invalidez con un incremento del $4.6 \%$ que equivale a 16 personas.

TABLA N ${ }^{\circ} 1$

ESTADISTICA DE RIESGOS PROFESIONALES CONSOLIDADO DE ADMINISTRADORAS DE RIESGOS PROFESIONALES QUE OPERAN EN COLOMBIA CLASE DE RIESGO V - SECTOR DE LA CONSTRUCCIÓN

\begin{tabular}{cc}
\hline $\mathbf{2 0 1 0}$ & $\mathbf{2 0 1 1}$ \\
\hline ENFERMEDAD & PROFESIONAL POR ACTIVIDAD \\
918 & 889 \\
$9.8 \%$ & $9.8 \%$
\end{tabular}

ACCIDENTALIDAD LABORAL

$\begin{array}{lc}73.461 & 109.726 \\ 16.6 \% & 20.1 \%\end{array}$

INCAPACIDAD PERMANENTE O PARCIAL

$\begin{array}{cc}1803 & 1864 \\ 18.7 \% & 18.7 \%\end{array}$

INVALIDEZ

$\begin{array}{ccc}46 & & 64 \\ 20.0 \% & & 24.6 \% \\ & \text { MORTALIDAD } & \\ & \text { Cont. Tabla No. 1 } \\ 2010 & & 2011 \\ 161 & 105 \\ 32.3 \% & 27.7 \%\end{array}$

COBERTURA DE EMPRESAS

Continuación de la Tabla

\begin{tabular}{cc}
51.245 & 61.854 \\
$11.4 \%$ & $12.6 \%$ \\
COBERTURA DE TRABAJADORES \\
933.403 & 1.169 .810 \\
$13.7 \%$ & $15.6 \%$ \\
\hline Fuente: CTRP - Fasecolda, encuesta sistema de información gremial, cá- \\
culo CTRP-Fasecolda
\end{tabular}

Según tasa de distribución geográfica el departamento del Quindío posee una tasa de accidentalidad del $7.0 \%$ con algunos casos de muerte (Positiva, 2012), la anterior cifra indica la existencia de situaciones y eventos que requieren de control para su respectiva solución, cifra importante para el desarrollo del presente trabajo. (Ver Tabla N²).

Estos reportes de accidentes se realizan a través de las administradores de riesgos laborales, quienes tienen identificados las diversas situaciones que aquejan al sector, estas entidades tienen la función de concientizar, capacitar, orientar, asistir a las empresas en el cumplimiento total del programa integral, pero queda como responsabilidad a la gerencia de estas, si dicho programa se ejecuta en su totalidad.

En relación al tema de seguridad y salud ocupacional, en la actualidad se evaden obligaciones y responsabilidades por parte de empleadores, esto se debe al poco interés por parte de la dirección en programar acciones, además la no disposición de inspección, vigilancia, control y seguimiento de los organismos o grupos responsables en el tema que les compete,

La siguiente tabla permite observar que en la mayoría de las ciudades existe un incremento de eventos de riesgo para los trabajadores en las diversas empresas, con un aumento de accidentalidad en ciudades como Vaupés, la Guajira, Chocó y Cesar.

Según informe continental año 2008 resultado de una 
encuesta nacional resume que estos reportes realizados al sistema general de riesgos profesionales se deben a una escasa asesoría por parte de estas administradoras de riesgos y al área administrativa por no contar con un programa de promoción y prevención adecuado y oportuno.

La accidentalidad laboral en el sector de la construcción reportada está relacionada con el cargo u ocupación, estos porcentajes están sujetos a la rotación de empleados, variedad de oficios, condiciones de tiempo y obviamente a la probabilidad del riesgo; encontrando que el $58.48 \%$ corresponde a obreros de la construcción, con un aporte del sector al PIB nacional del 3.6\% (Superintendencia de Sociedades, 2013).

Las consecuencias de esta probabilidad de riesgo son consideradas lesiones ocupacionales, las cuales son producidas por: negligencia en acatar responsabilidades estipuladas en los reglamentos internos o por el empleador, no disposición en asistir a capacitaciones, realizar actividades por parte del empleado por considerarlas que están bien ejecutadas aun reconociendo el riesgo, no uso de dotación e implementos de seguridad y demás altos inseguros que hacen de esta una actividad de alto riesgo.

TABLA No2

ESTADISTICA DE RIESGOS PROFESIONALES ACCIDENTE DE TRABAJO

SEGÚN DISTRIBUCIÓN GEOGRAFICA TASA DE ACCIDENTALIDAD

AÑO 2010 - 2011

\begin{tabular}{clcc}
\hline $\mathbf{N}^{\mathbf{0}}$ & \multicolumn{1}{c}{ CIUDAD } & $\mathbf{2 0 1 0}$ & $\mathbf{2 0 1 1}$ \\
\hline 1 & Amazonas & 2.1 & 3.1 \\
2 & Antioquía & 7.6 & 9.1 \\
3 & Arauca & 2.8 & 3.0 \\
4 & Atlántico & 7.8 & 7.7 \\
5 & Bogotá D.C. & 5.4 & 6.1 \\
6 & Bolívar & 8.4 & 8.3 \\
7 & Boyacá & 4.4 & 7.7 \\
8 & Caldas & 8.2 & 7.9 \\
9 & Caquetá & 4.5 & 5.3 \\
10 & Casanare & 4.5 & 5.3 \\
11 & Cauca & 7.3 & 7.1 \\
12 & Cesar & 12.0 & 10.1 \\
13 & Choco & 2.0 & 3.7 \\
14 & Córdoba & 5.2 & 6.2 \\
15 & Cundinamarca & 10.2 & 10.9 \\
16 & Guainía & 1.0 & 1.2
\end{tabular}

\begin{tabular}{clcc}
17 & Guaviare & 5.2 & 7.0 \\
18 & Huila & 3.8 & 5.2 \\
19 & La Guajira & 4.7 & 3.2 \\
20 & Magdalena & 10.2 & 9.8 \\
21 & Meta & 5.0 & 5.3 \\
22 & Nariño & 3.1 & 5.0 \\
23 & Norte de Santand. & 4.6 & 7.8 \\
24 & Putumayo & 1.7 & 2.5 \\
25 & Quindío & 5.6 & 7.0 \\
26 & Risaralda & 8.3 & 7.6 \\
27 & San Andrés y P & 6.2 & 8.2 \\
28 & Santander & 6.5 & 7.8 \\
29 & Sucre & 3.7 & 4.4 \\
30 & Tolima & 6.7 & 7.1 \\
31 & Valle & 6.9 & 7.9 \\
32 & Vaupés & 2.5 & 0.8 \\
33 & Vichada & 2.2 & 7.0 \\
\hline uente: CTRP - Fasecolda, encuesta sistema de información gre- \\
\hline ial, cálculo CTRP-Fasecolda & & \\
\hline
\end{tabular}

El tema en seguridad industrial y salud ocupacional tiene su sustento en leyes, normas, decretos aún vigentes (UGT, 2012), los cuales contiene disposiciones hacía la protección del trabajador y medidas sanitarias, contenidas en la política pública para la protección de la salud en el trabajo, con el fin de que las necesidades de los diferentes responsables sean atendidas y cumplidas. Actualmente se han realizado cambios previsibles en la norma técnica OHSAS 18001 la cual pasa a ser ISO 45001:2016 con el objeto de identificar riesgos y controles, que permiten que la empresa opere bajo condiciones mínimas básicas, esta norma puede ser certificable internacionalmente y se convierte en una ley interna. Los decretos 1443 de 2014 y el 1072 de 2015, reglamentan la ley 100 de 1993 y el decreto 1295 de 1994 en los artículos que competen a la reclasificación de empresas, la investigación del COPASST y al accidente de trabajo $\mathrm{y}$ enfermedad profesional con muerte del trabajador.

Esta normatividad legal, alienta a los empleadores y sus empleados, a la participación activa en la planeación estratégica y programación de actividades de promoción y prevención, que conforman el plan integral $\mathrm{SISO}^{2}$, este debe ser supervisado por organismos del estado y asesorados por las Entidades promotoras de riesgos laborales, con el fin de evitar consecuen-

\footnotetext{
2.Abreviatura que significa sistemas en seguridad y salud ocupacional.
} 
cias al trabajador y con incidencia para las empresas, generando gastos por incapacidades, servicios médicos y pérdida de ingresos.

Por lo tanto surge la siguiente interrogación:

¿En el Municipio de Armenia, las empresas constructoras y los profesionales gestionan el sistema integrado de salud ocupacional y seguridad industrial exigido para los proyectos de construcción?

No se conoce la existencia de investigaciones en Armenia, que permitan evaluar la aplicabilidad de planes integrales en el programa de seguridad industrial y salud ocupacional en las empresas; por lo tanto, se hace necesario la consulta de varios documentos y trabajos de investigación cuyos autores, destacan contribuciones y metodologías que permiten el análisis de las causas de accidentalidad y siniestros ocurridos en las diversas empresas como aporte al tema central de la investigación; Comentan: (Carvajal,2009; Asociación de seguridad, 2009; Cárdenas,2011), la necesidad inicialmente de realizar un análisis de accidentalidad, el cual permite determinar el impacto social y económico de los accidentes, sugiere como el uso una serie de formatos para que sean diligenciados y permitan determinar la causa posible del evento o suceso. -Establece un modelo a seguir para la sistematización de los procesos ejecutados, en tareas potencialmente peligrosas.

-Recomienda elaborar hoja de datos para establecer sugerencias en la política de salud y seguridad, con supervisión del programa e inspección de procedimientos, cambios de comportamiento y actitud del personal, desarrollo de programas de mejoramiento para la prevención de accidentes como medida para mitigar el riesgo laboral, ejercer control, supervisión e inspección, comprometer a la gerencia para brindar seguridad al trabajador.

(Universidad libre,2011; Suratep,2012; Carter, 2012), en sus documentos: establecen la urgencia de plantear programas de mejora para el sistema de vigilancia en el cumplimiento de las normas, sugiere que el personal calificado es importante para el desarrollo efectivo de programas y desempeño en las políticas de prevención, sugiere modelo de prevención de accidentes con el fin de reevaluar el plan actual mediante el planteamiento de objetivos, implementar acciones para condiciones seguras, manejo integral de accidentes, seguimiento a procesos internos y documentación de procesos de trabajo, mantenimiento de áreas y equipo e implementos de seguridad como dotación para acti- vidades de alto riesgo, programa software como sistema virtual para identificación de peligros y acciones de mejora en el manejo del riesgo. Este referente en su análisis indica que el sistema integral SISO no ha sido evaluado e investigado, por lo tanto se hace necesario con el aplicativo como encuesta calificar el grado de aplicabilidad de los programas en las empresas y aplicar la guía de control diseñada para una empresa seleccionada.

\section{DISEÑO METODOLOGICO}

El alcance geográfico de la investigación es el Municipio de Armenia, su desarrollo inicia identificando los proyectos del sector de la construcción de tipo vivienda, edificaciones y obras civiles.

El diagnóstico sobre la situación de la seguridad y salud ocupacional en estas empresas está representado por empresas constructoras, profesionales de la construcción, que tienen por obligación cumplir las disposiciones contenidas en el estatuto de seguridad industrial en resolución número 1016 de Marzo 31 de 1989.

La investigación se basa en el Decreto 1295 de Junio 22 de 1994 específicamente en el capítulo VI, correspondiente a la promoción y prevención de riesgos laborales, en el cual responsabiliza y obliga al patrono a ofrecer condiciones y ambientes seguros al personal que labora en las empresa para que la ejecución de las tareas sea adecuada y satisfactoriamente.

La promoción y prevención de riesgos laborales plantea para las empresas la ejecución de un plan integral que ayuda y facilita en las diversas áreas de las mismas a identificar las situaciones, eventos de riesgo, sus causas y consecuencias, es considerado como un ejercicio dinámico que puede ser ajustado de acuerdo a las necesidades de cada una de las empresas que conforman esta actividad económica específicamente.

El anterior sistema está compuesto por una serie de programas, los cuales poseen una estructura muy completa y en línea, con un cubrimiento total de la organización en la detección del riesgo, permitiendo una evaluación periódica, de tal manera que las medidas preventivas y/o correctivas sugeridas a través de informes finales sean aplicados oportunamente, con políticas de seguimiento. 
Estos servicios especializados son considerados un soporte eficaz en la planeación, prevención y evaluación organizacional, con la ayuda de informes finales establece y sugiere criterios y prioridades a diversos aspectos que requieren solución rápida.

La inexistencia de información sobre el tema propuesto, permite definir acciones urgentes que faciliten adquirir bibliografía, cifras, referentes estadísticos e historia general de la seguridad y salud de los trabajadores, para el desarrollo final de la investigación.

Teniendo en cuenta lo antes mencionado, se realiza un diagrama de flujo detallando las actividades esenciales que permiten en primera instancia explicar información obtenida, cálculos realizados, análisis y resultados alcanzados mediante métodos y técnicas que facilitan el desarrollo del tema propuesto. (Ver Diagrama No. 1)

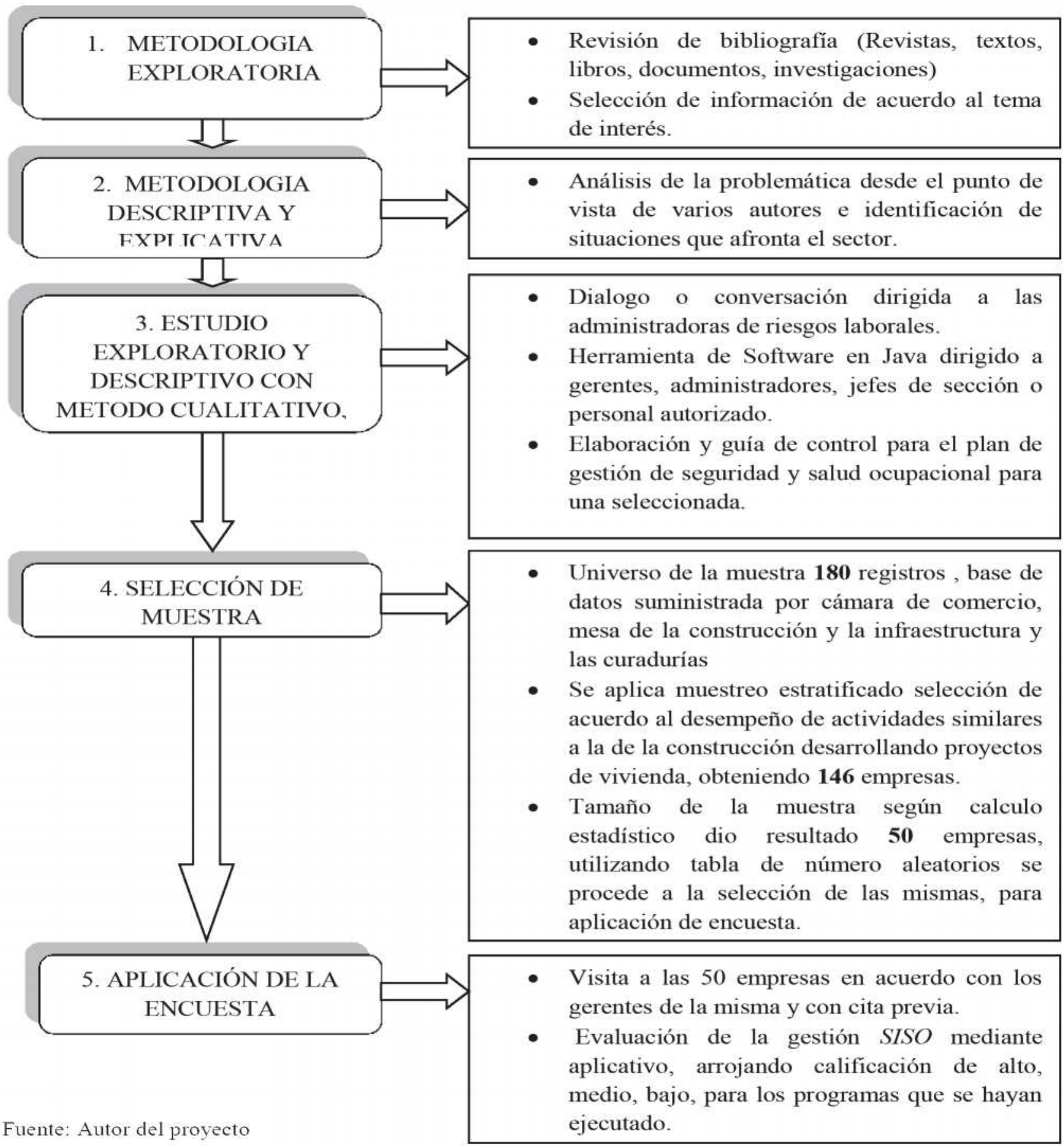

DIAGRAMA Nº1 DISEÑO METODOLOGICO 
La metodología aplicada es la exploratoria inicialmente en forma cualitativa, ya que no existe información alguna sobre estudios realizados en lo que respecta a la aplicabilidad de los planes integrales siso; por lo tanto esta investigación facilita recoger e identificar aspectos puntuales que permitan conocer la problemática planteada según punto de vista de diversos autores con el apoyo de revistas, textos, investigaciones y documentos, sobre la identificación de situaciones que afronta el sector, en lo que concierne a aspectos de seguridad social y salud ocupacional (OIT,2005), facilitando un análisis de las causas de los fenómenos según condición del evento, como también la interpretación cuantitativa en la aplicación de tabulaciones, formulas, gráficas, además del método como instrumento aplicativo de software en java, para la recolección de información en campo y determinar situación actual de las empresas para su respectivo diagnóstico.

El universo de la muestra son todas aquellas empresas constructoras registradas en la base de datos suministrada mediante oficinas de cámara de comercio (2014), mesa de la construcción y la infraestructura (2014), curadurías $\mathrm{N}^{0} 1$ y No2(2014), que realicen actividades similares a la de la construcción desarrollando proyectos de vivienda en el sector, la base de datos obtenida contiene 180 registros de empresas que permiten ser ordenados de menor a mayor y clasificados por actividad y número de trabajadores, se "aplica la técnica de muestreo estratificado ${ }^{3 "}$, obteniendo como resultado del filtro 146 empresas, las cuales serán tenidas en cuenta para el cálculo estadístico y poder establecer así el número de empresas a seleccionar para aplicación de la encuesta.

Conociendo el tamaño de la muestra se procede a aplicar el muestreo aleatorio, utilizando la tabla de números aleatorios. La visita a estas se realiza para identificar los aspectos más relevantes de la gestión SISO arrojando datos estadísticos sobre aspectos de gestión en salud ocupacional y seguridad industrial establecidos en la escala básica, avanzada, especializada, gestión integral, con indicadores de calificación así: alto, medio, bajo.

También aplica el estudio exploratorio y descriptivo,

\footnotetext{
${ }^{3}$ Consiste en separar la población de estudio en grupos o sectores respecto a la característica a estudiar, dando como resultado un determinado número de miembros, que serán quienes conformen la muestra.
}

con método cualitativo, cuantitativo en aplicación de técnicas de sondeo de opinión (fuentes institucionales) y test aplicativo construido en Java; estructurados así:

-Dialogo o conversación dirigida a las Administradoras de Riesgos Profesionales.

-Herramienta de Software en Java dirigido a gerentes, administradores, jefes de sección o personal autorizado, con el objeto de obtener información referente a la aplicabilidad del plan SISO en cada empresa.

Finalmente, mediante metodología de gestión integral de proyectos se elabora una guía de control para el plan de gestión de seguridad y salud ocupacional, para una empresa que el proyecto considere necesario y urgente implementar, describir y estructurar políticas, planes, procedimientos, operaciones, acciones correctivas y revisiones respectivas con el objeto de mejorar sus procesos.

\section{ANALISIS DE RESULTADOS}

\section{Recolección de información}

-La herramienta utilizada es un instructivo que consta de 6 secciones, el cual permite evaluar la situación actual de la empresa, dirigida a las empresas del sector de la construcción según listado obtenido de acuerdo a la selección números aleatorios, específicamente a la sección o área de salud ocupacional del proyecto, mediante cita previa con autorización de la gerencia, aplicativo que tuvo una duración de 1 hora aproximadamente, a través de formato estructurado y sistematizado.(ver Anexo $\mathrm{N}^{\circ} 1$ ).

\section{Sistematización}

-Construcción tabla de datos calificación obtenida. (ver Tabla $\mathrm{N}^{\circ} 3$ ).

-Elaboración de figuras teniendo en cuenta los ítems más significativos de la encuesta para su correspondiente interpretación.

\section{Resultados}

La información recolectada corresponde a 44 formularios y test diligenciados, los cuales equivalen a un $88 \%$, con un error muestral de 0.04 y un nivel de confianza del $95 \%$ del total equivalen a un $88 \%$, con un error muestral de 0.04 y un nivel de confianza del 
95\% del total de 50 empresas a encuestar según cálculo del tamaño de la muestra.

Los datos obtenidos permiten el siguiente análisis:

\section{Porcentaje de aplicación de programas}

Para la aplicación de programas se clasifican las empresas constructoras y a los ingenieros, arquitectos constructores a quien se denomina constructores particulares, graficas las cuales contienen los porcentajes de calificación para cada uno de los programas y su respectiva evaluación.(ver-figuras).

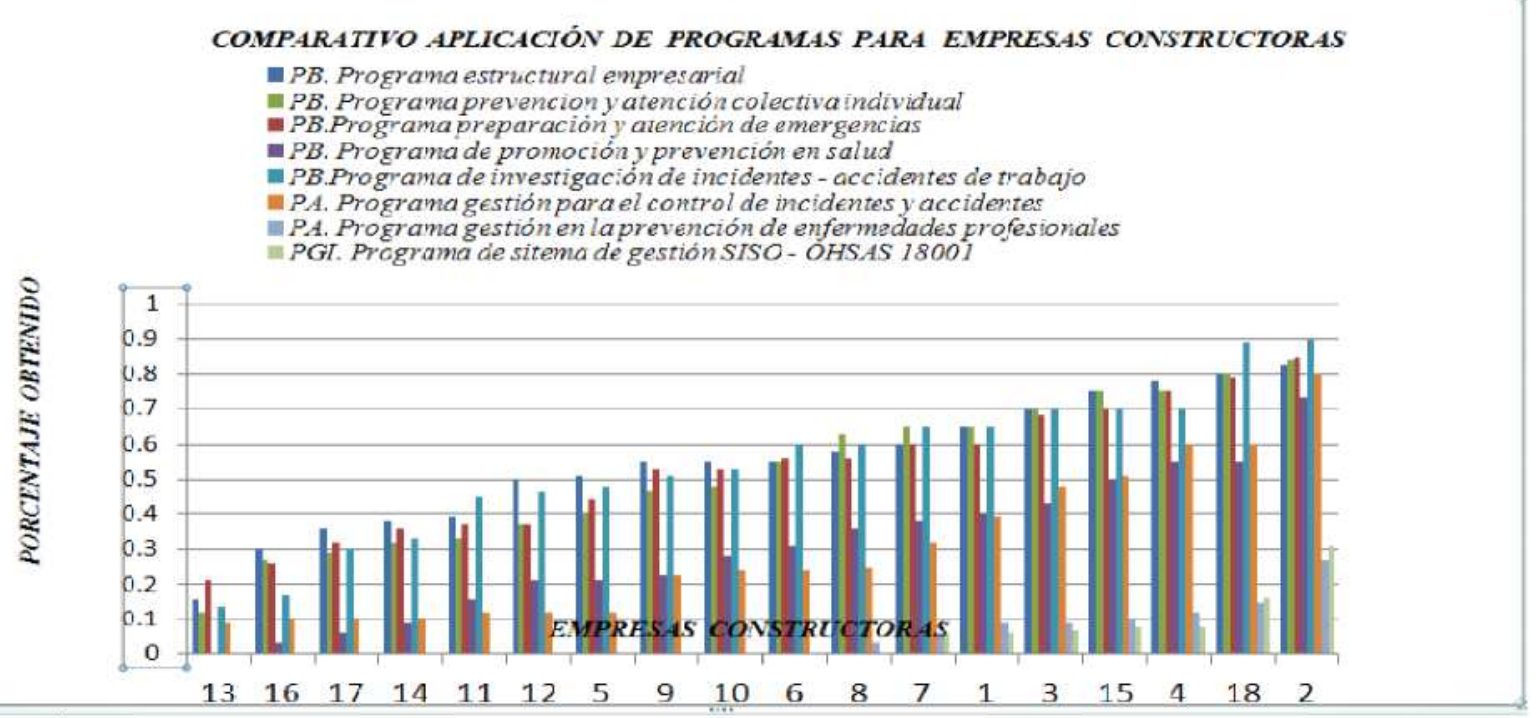

Fuente: Autor proyecto

FIGURA N $\mathbf{N}^{0}$ 1. Aplicación programas empresas constructoras.

Compara el número de programas ejecutados por cada una de las empresas.

Son empresas reconocidas en el municipio, con trayectoria en el desarrollo de proyectos de vivienda, constituidas legalmente y con una estructura empresarial.

Se encuestaron 18 empresas constructoras que equivalen a un $36 \%$ de las 44 encuestas realizadas.

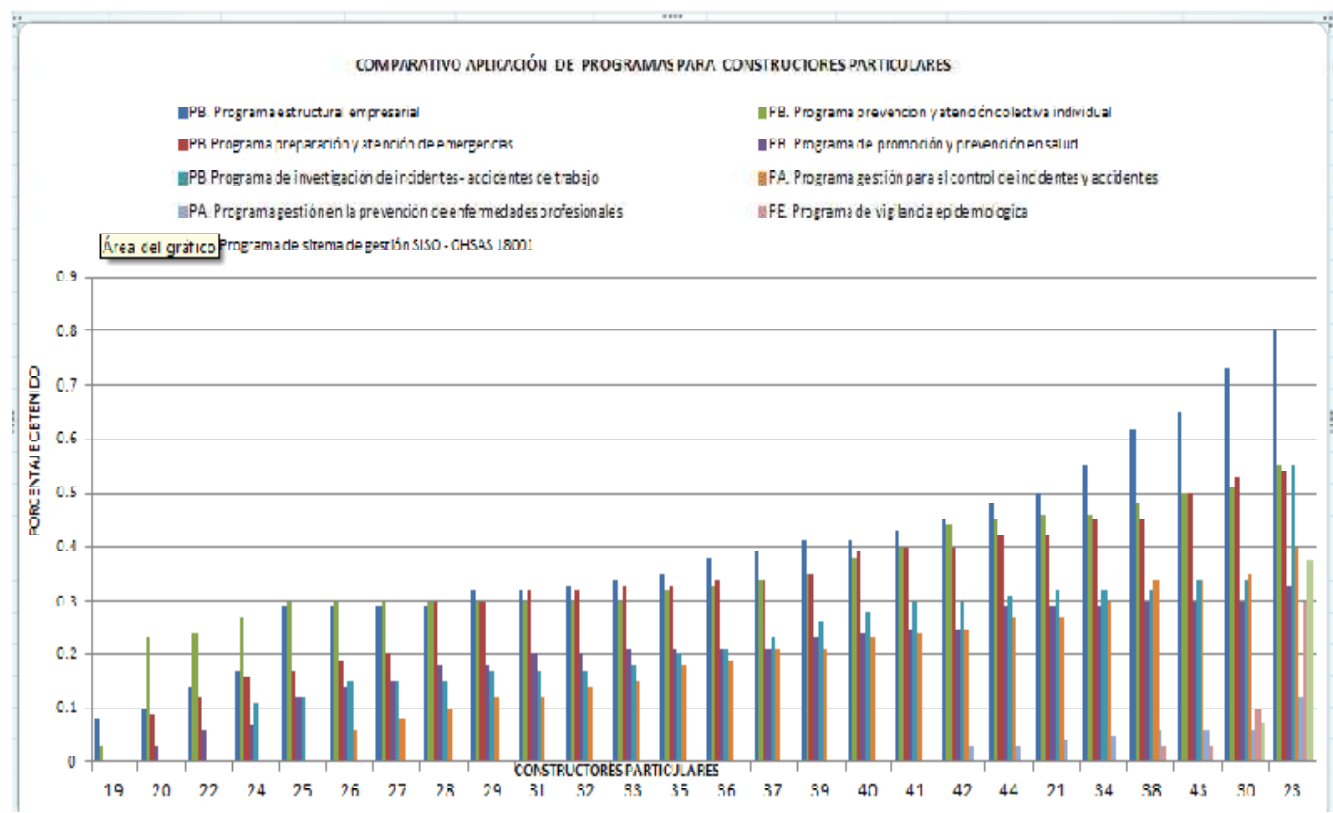

Fuente: Autor proyecto

FIGURA N $\mathbf{N}^{\mathbf{2}} \mathbf{2}$. Aplicación programas para constructores particulares.

Compara el número de programas ejecutados por cada una de los ingenieros o gerentes de obra. 
El grupo de constructores particulares lo conforman ingenieros y arquitectos gerentes y administradores de proyectos de vivienda, con reconocimiento a nivel municipal, además de dirigir obras a lo largo del departamento. Se encuentran registrados como empresas constituidas.

Se realizaron 26 encuestas equivalente al $47.27 \%$ del total diligenciado.

La figura $\mathrm{N}^{\circ} 1$ y $\mathrm{N}^{0} 2$ muestran que empresas constructoras y los constructores particulares tienen desarrollado en algún grado los programas en salud ocupacional y seguridad industrial, por lo tanto emite el siguiente análisis:

Teniendo en cuenta que son 9 programas se les asigna una nomenclatura para su identificación así:
1-PEE: Programa estructural empresarial

2-PPACI: Programa de prevención y atención colectiva individual

3-PPAE: Programa de atención y preparación de emergencias

4-PPPS: Programa de promoción y prevención en salud

5-PIIAT: Programa de investigación de incidentes a accidentes de trabajo

6-PGCIA: Programa gestión para el control de incidentes y accidentes

7-PGPEP: Programa gestión en la prevención de enfermedades profesionales

8-PVE: Programa de vigilancia epidemiológica

9-PSGSISO: Programa de sistema de gestión SISOOHSAS 18001

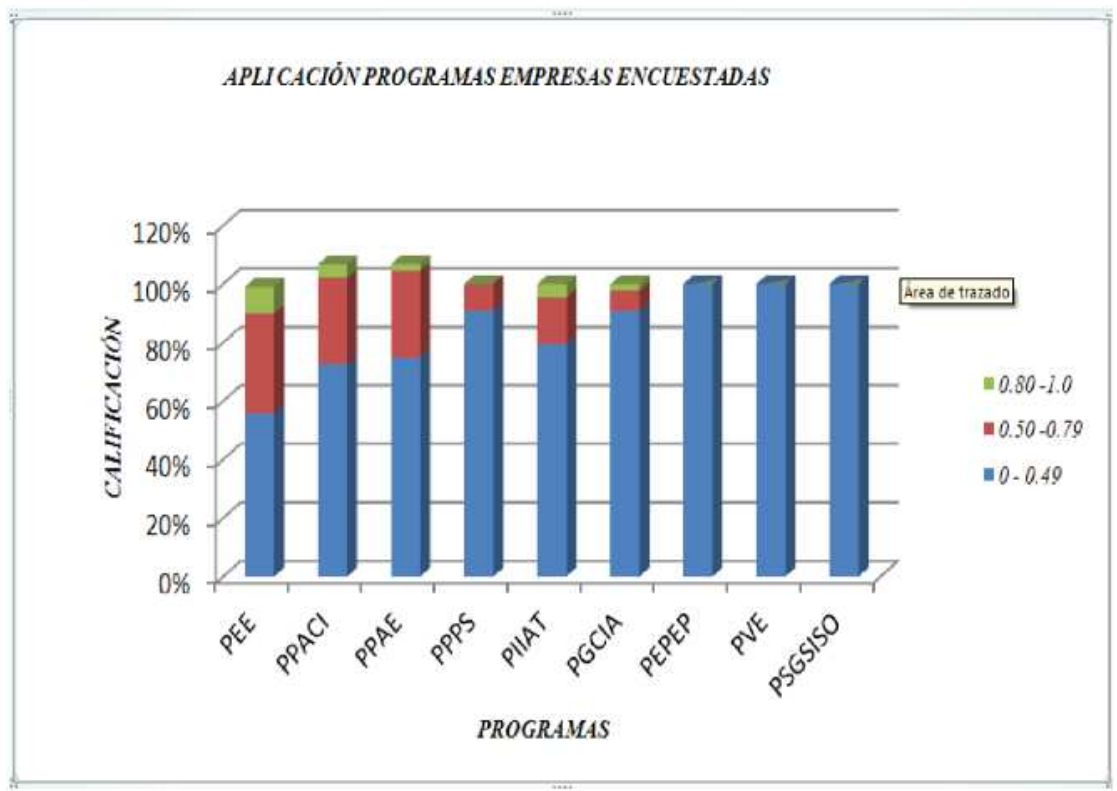

Fuente: Autor proyecto.

FIGURA No 3. Aplicación programas empresas encuestadas. El eje X representa los programas que compone el sistema general en salud Ocupacional y seguridad industrial y el eje $\mathrm{Y}$ el valor porcentual de calificaciónSegún grado de ejecución del programa.

A continuación se presentan los datos obtenidos para la evaluación de los programas que las empresas del sector de la construcción tienen ejecutados con su respectiva calificación, los programas aquí enumerados hacen parte y/o conforman el sistema integral de salud ocupacional y seguridad industrial que toda em- presa debe desarrollar para proporcionar al empleado condiciones seguras de trabajo, lo que proporciona al empleador inmensa satisfacción por dar cumplimiento de lo establecido por la ley, y así evitar y prevenir situaciones de riesgo en las diversas áreas de la empresa. 
TABLA N³. Resultados de evaluación en la aplicación de programas para empresas encuestadas.

\begin{tabular}{|c|c|c|c|c|c|c|c|}
\hline \multirow[t]{2}{*}{ PROGRAMAS } & \multicolumn{3}{|c|}{$\begin{array}{c}\text { RANGO DE } \\
\text { CALIFICACIÓN }\end{array}$} & \multicolumn{3}{|c|}{$\begin{array}{l}\text { PORCENTAJE DE } \\
\text { CALIFICACIÓN }\end{array}$} & \multirow{2}{*}{$\begin{array}{c}\text { TOTAL } \\
\text { ENCUESTAS } \\
\text { NUMERO }\end{array}$} \\
\hline & 0-0.49 & 0.5-0.79 & $0.8-1.0$ & ВАJO & MEDIO & ALTO & \\
\hline $\begin{array}{l}\text { 1.PEE (Programa Estructural } \\
\text { Empresarial) }\end{array}$ & 25 & 15 & 4 & 56 & 34 & 9.09 & 44 \\
\hline $\begin{array}{l}\text { 2.PPAE (Programa de prepa- } \\
\text { ración y atención de emergen- } \\
\text { cias) }\end{array}$ & 30 & 13 & 1 & 75 & 29.54 & 2.27 & 44 \\
\hline $\begin{array}{l}\text { 3. PPACI (Programa de pre- } \\
\text { vención y protección colectiva } \\
\text { e individual }\end{array}$ & 32 & 10 & 2 & 72.7 & 22.7 & 4.5 & 44 \\
\hline $\begin{array}{l}\text { 4. PPPS (Programa de promo- } \\
\text { ción y prevención en salud) }\end{array}$ & 40 & 4 & 0 & 90.9 & 9.1 & 0 & 44 \\
\hline $\begin{array}{l}\text { 5. PIIAT (Programa de } \\
\text { investigación de incidentes y } \\
\text { accidentes de trabajo) }\end{array}$ & 35 & 7 & 2 & 79.5 & 15.9 & 4.54 & 44 \\
\hline $\begin{array}{l}\text { 6. PGCIA (Programa de } \\
\text { gestión para el control de } \\
\text { incidentes y accidentes) }\end{array}$ & 40 & 3 & 1 & 90.9 & 6.80 & 2.27 & 44 \\
\hline $\begin{array}{l}\text { 7. PGPEP (Programa de } \\
\text { gestión en la prevención de } \\
\text { enfermedades profesionales) }\end{array}$ & 44 & 0 & 0 & 100 & 0 & 0 & 44 \\
\hline $\begin{array}{l}\text { 8. PVE (Programa de vigilan- } \\
\text { cia epidemiológica) }\end{array}$ & 44 & 0 & 0 & 100 & 0 & 0 & 44 \\
\hline $\begin{array}{l}\text { 9. PSGSISO (Programa del } \\
\text { sistema de gestión SISO- } \\
\text { OHSAS } 18001 \text { ) }\end{array}$ & 44 & 0 & 0 & 100 & 0 & 0 & 44 \\
\hline
\end{tabular}

La tabla anterior permite realizar el siguiente análisis: -PEE: Programa estructural empresarial con rango de calificación entre:

$0-0.49$ (25 empresas), $0.5-0.79$ (15 empresas) y de 0.8 - 1.0 (4 empresas). Las empresas evaluadas con una calificación media corresponden al $34 \%$, bajo al $56 \%$ y alto al $9.09 \%$ de las 44 encuestas realizadas, entendiendo con ello que algunas actividades no se terminan y otras no se ejecutan, por lo tanto no existe un eficaz direccionamiento estratégico en prevención y promoción del riesgo, además no han identificado como mínimo las condiciones de peligro en el ambiente laboral y no han realizado planes de acción como correctivos.

-PPAE: Programa de preparación y atención de emergencias con rango de calificación entre:0 -0.49 (30 empresas), $0.5-0.79$ (13 empresas) y de $0.8-1.0$ (1 empresa). El mayor porcentaje es de $75 \%$ con empresas que se encuentran dentro del rango bajo, lo cual indica que solo existe un leve inicio de las actividades a desarrollar, pero no hay un total cumplimiento de las estrategias establecidas en caso de actuar ante un desastre o amenaza presentes o aun potenciales, indica también la no existencia de procedimientos, protocolos, entrenamientos y formación ante las situaciones que se puedan generar por ocasión de accidentes, concertación de rutas a seguir en caso de desastre o amenaza.

-PPACI: Programa de prevención y protección colectiva e individual; su calificación con un rango de: 0 0.49 (32 empresas), $0.5-0.79$ (10 empresas) y de 0.8 -1.0 (2 empresas). Para el rango bajo 32 empresas, muestra el poco compromiso por parte del empleador en brindar al empleado las condiciones y sitios de trabajo seguros para desempeño de actividades, correspondiendo en porcentaje a un $72.7 \%$, empresas que no han establecido procedimientos de trabajo seguro, medidas de protección y cuidado en caso de ser afectados por ocurrencia de eventos que desencadene situaciones de peligro en el desempeño de las actividades. 
-PPPS: Programa de promoción y prevención en salud con rango entre: $0-0.49$ (40 empresas), $0.5-$ 0.79 ( 4 empresas) y de $0.8-1.0$ (o empresas), correspondiendo a $90.9 \%$ para 40 empresas del sector. Este porcentaje evidencia la falta de información de la alta gerencia en no tener como mínimo base de datos de la población trabajadora y situación médica en caso de ser expuesta a situaciones de riesgo.

A partir de este programa la calificación para los siguientes no es significativa. Las empresas en su mayoría obtienen calificación de cero, por lo tanto se puede decir no existen diagnósticos de enfermedad profesional, sistemas de vigilancia epidemiológica, ni políticas de calidad que redunden en el bienestar de los empleados y en el desempeño de las empresas, los porcentajes que corresponden a estos programas son: Para PGPEP, PVE, PSGSISO es de $100 \%$ para las 44 empresas con calificación baja, algunas empresas evaden las responsabilidades y obligaciones adquiridas en la contratación de sus empleados y han desarrollado sólo instrucciones básicas en lo que a seguridad industrial y salud ocupacional se refiere, no hay una sensibilización y concientización ante riesgos potenciales y poco compromiso en preservar las condiciones de vida y salud de los trabajadores

\section{Criterio de calificación cuantitativa}

La calificación cuantitativa permite dar a conocer el número de empresas que se acogen a las condiciones establecidas en la ejecución y el desarrollo de los programas que hacen parte del sistema SISO, el valor calificado como escala de puntaje, busca alertar al dueño de la empresa sobre la políticas y estrategias planteadas; para que estas sean reevaluadas, estudiadas, nuevamente aplicadas con el objeto de un mejoramiento continuo.

Esta calificación corresponde a la suma de cada uno de los porcentajes de calificación para los 9 programas que conforman el sistema de gestión en seguridad y salud ocupacional, ( la Figura $\mathrm{N}^{\circ} 4$ ), muestra que en las empresas constructoras, en relación a la meta estipulada, sólo 3 obtienen valores de 0.59 , 0.534 y 0.502 .

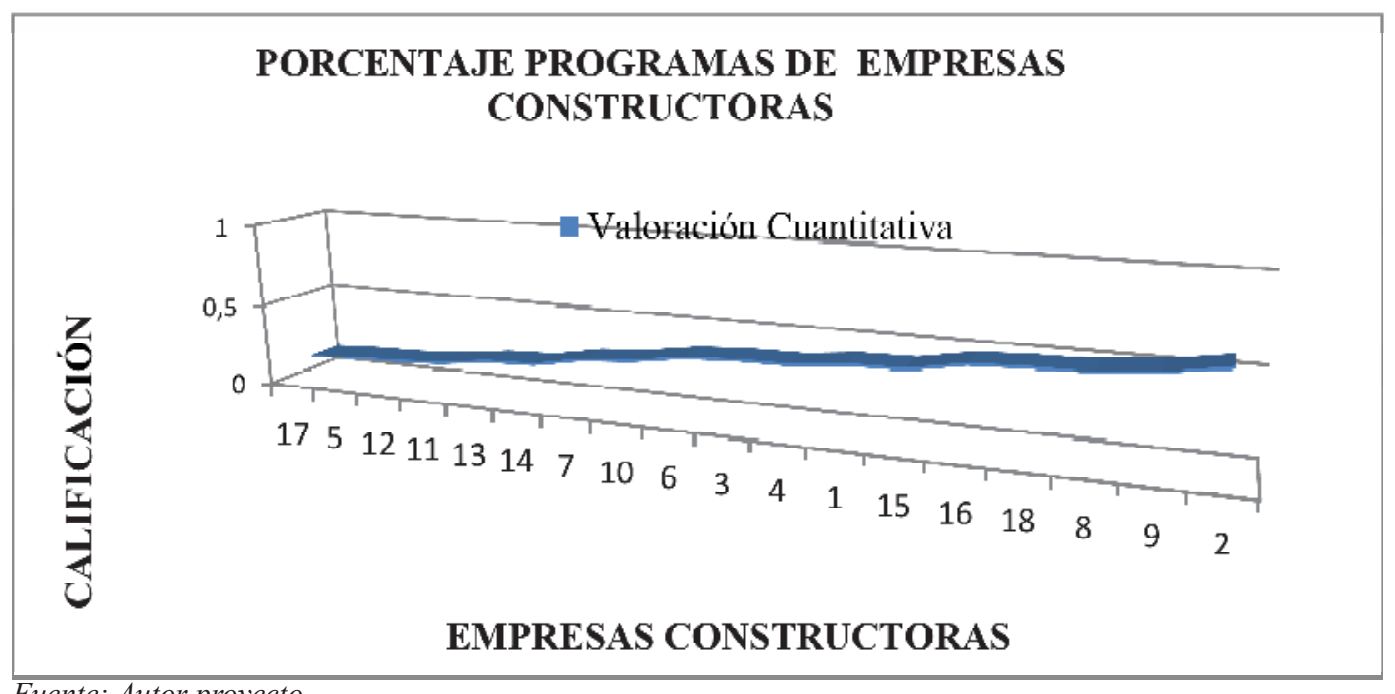

Fuente: Autor proyecto.

FIGURA 4. Porcentaje ejecutado de programas por empresas constructoras.

El eje $\mathrm{X}$ representa posición de empresas según programas ejecutados y el eje $\mathrm{Y}$ corresponde al valor porcentual calificado según programas ejecutados y desarrollados. 


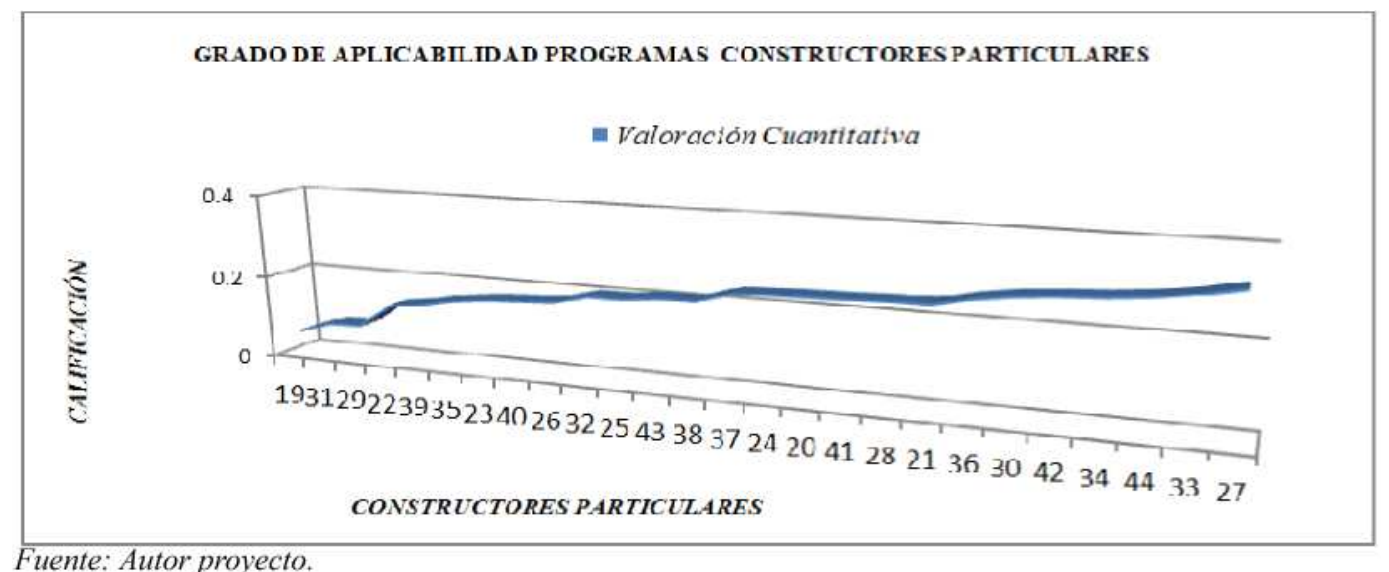

FIGURA N ${ }^{\circ}$ 5. Calificación cuantitativa constructores particulares

El eje X representa posición de constructores particulares según programas ejecutados y el eje Y corresponde al valor porcentual calificado según programas ejecutados Desarrollados.

Los máximos valores de calificación alcanzados es para 2 empresas con 0.3226 y 0.3064 respectivamente, lo que significa que aun teniendo algún porcentaje de calificación en los programas la suma global de ellos se encuentra en un rango bajo. (ver Figura $\mathrm{N}^{\circ} 5$ ).

\section{Criterio de calificación cualitativo}

Esta calificación permite informar a la empresa el grado de desarrollo y /o aplicación del sistema de gestión SISO, asignando de acuerdo al rango de calificación con un nivel de bajo, medio o alto. (ver Figura $\mathrm{N}^{\circ} 6$ ).

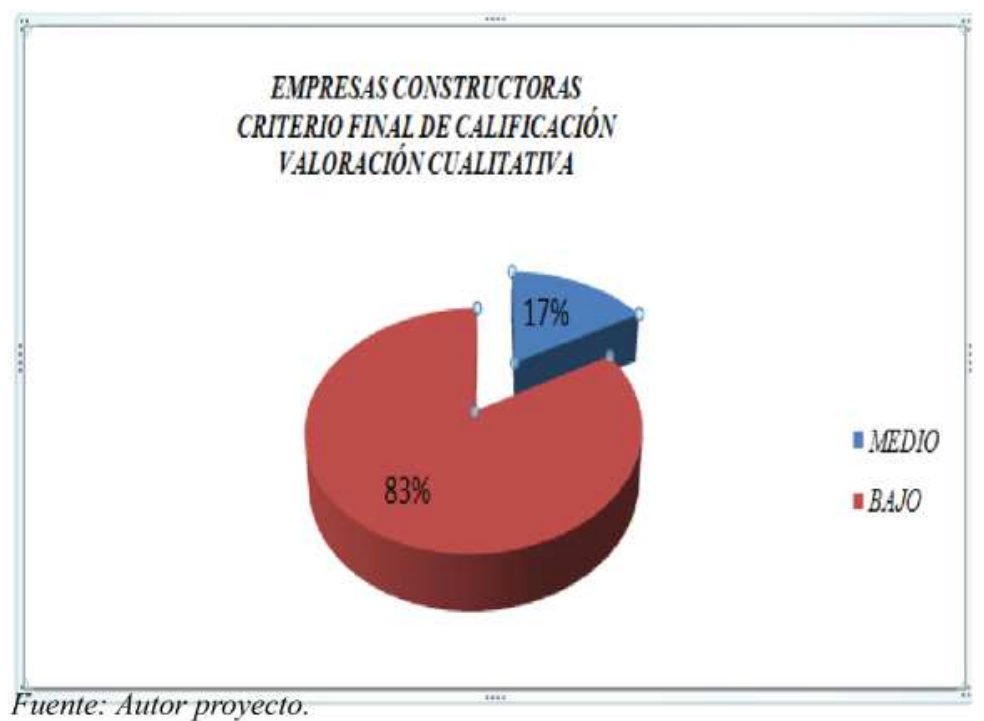

FIGURA N ${ }^{\circ}$ 6. Calificación cualitativa empresas constructoras.

Nivel de desarrollo de los programas que conforman el plan integral de salud Ocupacional y seguridad industrial para las empresas constructoras en el Municipio de Armenia.

Para las empresas constructoras el criterio de calificación en cuanto a valoración cualitativa corresponde: con un $83 \%$ a 15 empresas constructoras con grado de desarrollo bajo y grado medio con un porcentaje del 17\% para 3 empresas del sector; lo anterior indica que las empresas no han aprovechado los medios existentes como acompañamiento de entidades promotoras, asesoras y personal idóneo, sólo se han limitado hacer desde su conocimiento y experiencia el desarrollo de programas sin obtener resultados exitosos por considerar que se incurren en gastos económicos que no están contemplados en los presupuestos de obra. 


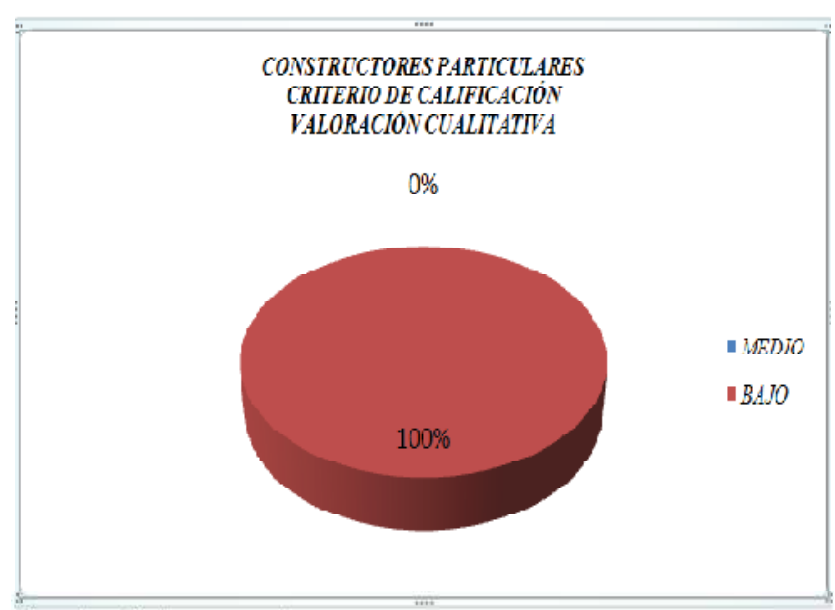

Fuente: Autor proyecto.

FIGURA N $\mathbf{N}^{\mathbf{7}}$ 7. Calificación cualitativa constructores particulares. Nivel de desarrollo de los programas que conforman el plan integral de salud ocupacional y seguridad industrial para los constructores particulares en el Municipio de Armenia.

Para los constructores particulares (ver Figura $\mathrm{N}^{\circ} 7$ ), el grado desarrollo de los programas obtuvo una ca- lificación de bajo, el cual corresponde al 100\% para 26 encuestas, porcentaje significativo y preocupante muestra negligencia por parte de los profesionales del sector ante normas establecidas, responsabilidades y obligaciones adquiridas con respecto al bienestar de sus empleados.

Estos criterios de evaluación de la gestión en seguridad y salud ocupacional, basa su evaluación en cuatro criterios técnicos del plan integral en cada empresa de acuerdo al programa ejecutado con un puntaje establecido permite calificarlo si este se cumple completamente, parcialmente, mínimo o no cumple.

\section{Acciones a realizar}

Ítem considerado de gran importancia para el trabajo porque permite de manera gráfica observar para cada uno de los 9 programas la sugerencia de acción a realizar dependiendo el estado en que se encuentre el programa. (Ver Figura $\mathrm{N}^{\circ} 8$ ).

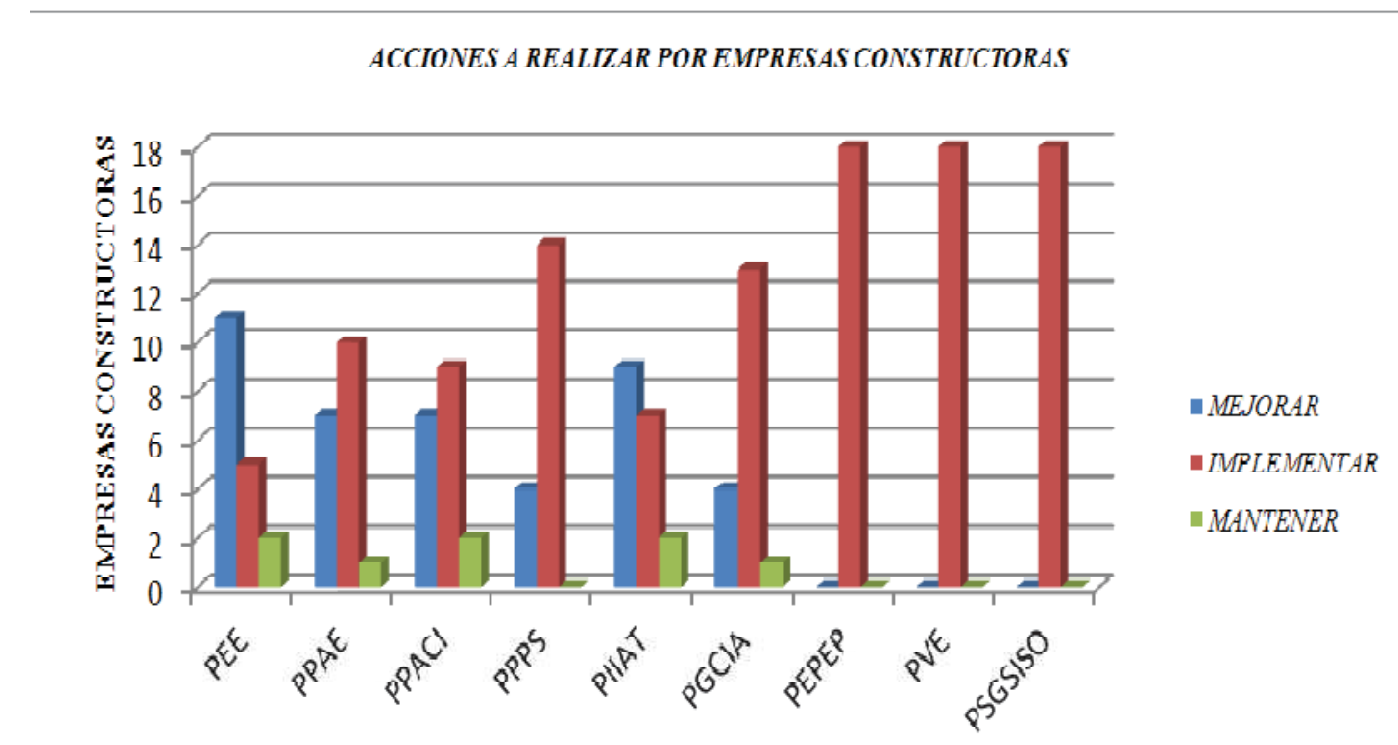

SUGERENCIAAPROGRAMAS

Fuente: Autor proyecto

FIGURA N $\mathbf{N}^{\mathbf{0}}$. Acciones a realizar por empresas constructoras.

El eje X representa los programas con grado de ejecución y en el eje Y se encuentran localizadas el número de empresas que aplican dichos programas.

La acción a realizar para estas empresas constructoras está determinada por la calificación inicial del programa que el instructivo asigna según grado de desarrollo del mismo, esta calificación es asignada en porcentaje según los siguientes rangos:

De 0 a 49\%: Implementar, 50 a 79\%: Mejorar, 80 a $100 \%$ : Mantener.
Por lo tanto para las empresas constructoras se recomienda:

Implementar para todos los programas una política de seguridad que permita en algún momento reevaluar procesos para cumplimiento de objetivos y metas trazadas. 
Mejorar para 6 programas: (5 básico) y (1 avanzado), con cierto grado de desarrollo, sugiere efectuar cambios, modificaciones y ajustes con el fin de buscar estándares en procesos.

Mantener para 5 programas (básico 4 y avanzado 1 ). a través de procedimientos con un control y seguimiento constante para garantizar efectividad en los procesos.

\section{ACCIONES A REALIZAR POR CONSTRUCTORES PARTICULARES}

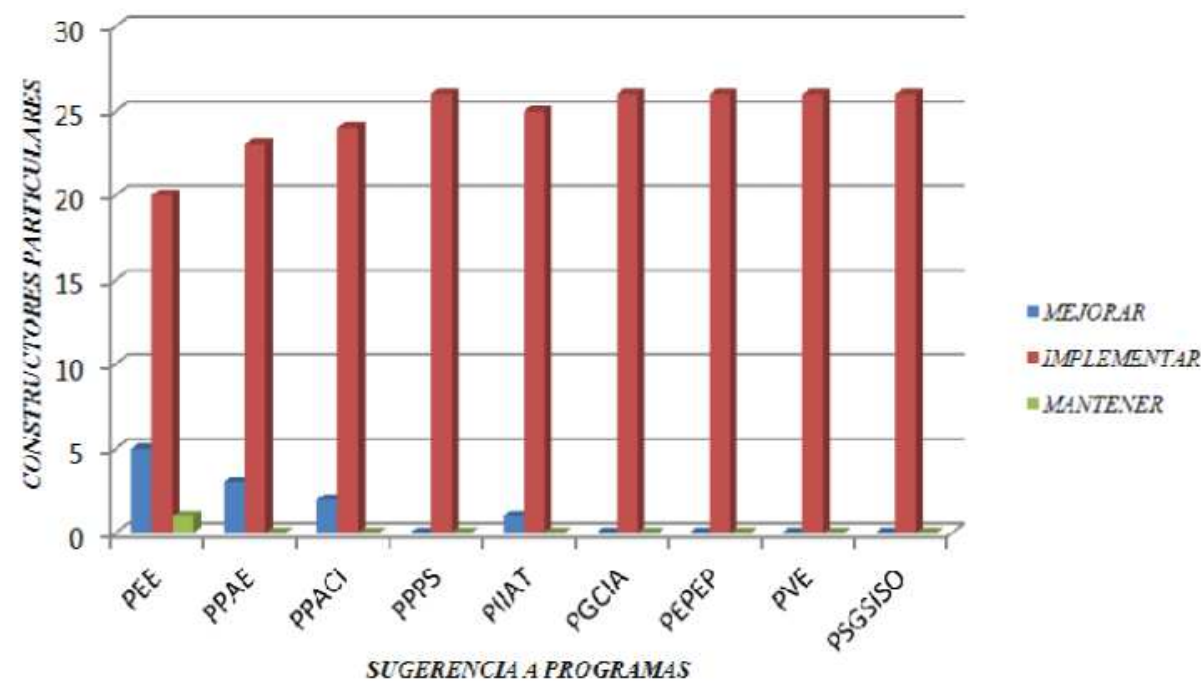

Fuente: Autor proyecto

FIGURA $\mathbf{N}^{0}$ 9. Acciones a realizar por constructores particulares.

El eje X representa los programas con grado de ejecución y en el eje Y se encuentran localizadas el número de empresas que aplican dichos programas.

En estas empresas la estrategia de prevención e intervención debe estar dirigida a la disminución de la probabilidad de ocurrencia de accidentes en áreas de trabajo.

Los planes básico, avanzado y especializado contienen una programación establecida para cada una de las actividades que permite aplicar medidas en la estructura empresarial, con el objeto de mejorar condiciones y evitar riesgos.

Estos riesgos generan resultados negativos para el sector, las consecuencias por daños a material, equipo, maquinaría e instalaciones y afecciones al personal son de gran costo económico y es la evidencia del no cumplimiento de una ley establecida que propone prevenir, disminuir y mitigar accidentes.

Para los constructores particulares esta calificación es un llamado de atención en el desarrollo de los 9 programas, recomienda implementar decisiones de gestión en la programación de una política en seguridad industrial teniendo en cuenta aspectos que con- tribuyan a mejorar desempeños y mantener procesos, procedimientos, actitudes hacía un mejoramiento continuo, apoyado de indicadores que permitan un seguimiento y control en todas las áreas del proceso.

\section{CONCLUSIONES}

Los datos obtenidos resultado del aplicativo arrojan el siguiente análisis:

Las empresas constructoras han ejecutado con algún porcentaje 6 programas que corresponden 5 al programa básico y 1 al avanzado del total de 9 que deben ejecutar, entre tanto los constructores particulares obtienen su calificación para los 3 primeros programas del básico.

Se debe tener en cuenta que ni las empresas constructoras, ni los constructores particulares han ejecutado algún programa en su totalidad.

Los porcentajes de calificación máxima se han obtenido en un rango de $0.8-0.9$ en programas de nivel 
básico, pero estos se han visto disminuidos por la calificación en los programas avanzado, especializado y de gestión, donde ha sido cero en valor y por lo tanto el programa tiene un criterio global de calificación en donde integra la calificación de todos los programas.

El grado de desarrollo de la gestión SISO para las 44 encuestas realizadas (las cuales fueron suficientes para establecer la situación actual), evidencia que el $17 \%$ ( 3 empresas) de estas obtuvo un rango de calificación media y 41 empresas restantes obtienen una calificación de bajo, por lo tanto se puede decir que para Municipio de Armenia los proyectos de vivienda no poseen políticas de salud ocupacional que ofrezcan a sus empleados ambientes y condiciones seguras de trabajo.

En cuanto a acciones a realizar tanto para empresas constructoras como para constructores particulares en todos los programas la más importante es la de implementarlos. Es un llamado de atención para que pongan en marcha aquellos programas establecidos y que estos permitan realizar cambios y mejoras hacía un mejoramiento continuo con el ánimo de estandarizar procesos, actividades que permitan mantener procedimientos y seguir su correcta aplicación.

En el diligenciamiento del aplicativo se pudieron detectar las siguientes situaciones:

Falta de conocimiento de los profesionales de la construcción involucrados en los procesos en lo que concierne al programa de seguridad siso ajustados a la normatividad vigente de manera que se hace urgente que en los pensum de formación académica se involucren asignaturas de esta referencia para que en el tiempo se generen los conocimientos necesarios para poner en práctica la legislación vigente.La poca claridad en cuanto a la documentación, trazabilidad y evidencias que se deben consignar en lo referente a temas siso en el desarrollo de la obra.
Se percibe que las empresas constructoras y constructores particulares creen salvar sus responsabilidades solo afiliando a sus empleados al sistema general de riesgos.

La guía de control diseñada y facilitada, es un aporte para aquellas empresas que no tienen programas establecidos en seguridad industrial y salud ocupacional. Además es una herramienta que permite el manejo del tema a través del seguimiento en cada uno de los ítems propuestos, con el fin de facilitar el manejo, control del riesgo y crear conciencia en fortalecer procesos y actividades en pro de una gestión en siso exitosa.

\section{RECOMENDACIONES}

Las empresas constructoras y constructores particulares deben asignar rubros para asesorías en seguridad industrial y salud ocupacional contemplados en los presupuestos de obra, con el fin de contratar al personal idóneo que ejecute programas y se pueden tener mejores resultados de gestión.

El estado debe reevaluar la función de los órganos de control en el desarrollo de programas imponer medidas al sector de la construcción que permitan el cumplimiento de los requisitos estipulados en la Ley para mejorar condiciones de trabajo en materia de seguridad industrial y salud ocupacional.

Las administradoras de riesgos laborales deben establecer campañas de sensibilización efectivas donde el sector de la construcción se capacite y asesore sobre los programas del sistema de gestión como solución a los índices elevados de accidentalidad.

Debe establecerse un sistema de gestión integral por parte de las empresas con énfasis en el recurso humano reconociendo que el personal integrante es el componente más importante en la organización y al cual se le deben brindar buenas condiciones de trabajo y salud laboral 


\section{BIBLIOGRAFÍA}

- Adecuación estructural. 2012. Informes estadísticos de empresas constructoras. Armenia. Curadurías $\mathrm{N}^{\circ} 1$ y $\mathrm{N}^{\circ} 2$. Municipio de Armenia.

- Alburquerque P, Walter. 2007. Identificación de peligros, evaluación y control de riesgos. Piura.

- Al-humaidi,HM. Hadipriano, TF. 2004. Safety construction in Kuwait, doi. 70-77p.

- Áreas secretarías y Salud Ocupacional. 2012. Reporte accidentes laborales empresas afiliadas. ARPS Municipio de Armenia.

- Asociación Colombiana de Seguros. 2010. Seguridad en colombia. Bogotá. Fasecolda.

- Association security of Ontario. 2009. A guide to developing health and safety policies and programs in construction. 87-88p.

- Betancur, F.M. 2005. Salud ocupacional un enfoque humanista: Como gerenciar la salud y la seguridad mediante el estimulo del auto cuidado y la autogestión. Bogotá, D.C. Editorial McGraw-Hill. Interamericana, S.A. $141 \mathrm{p}$.

- Asociación Colombiana de Seguros. 2010. Seguridad en colombia. Bogotá. Fasecolda

- Cárdenas Grisales, Paola Milena. 2011. Evaluación y análisis de las prácticas en seguridad industrial y salud ocupacional en empresas de construcción en Colombia. Tesis maestría. Bogotá, D,C. Universidad de los Andes. 89p.

- Carter, Gregory. Smith, Simón. 2012. It tool for construction site safety management.

- Carvajal Peláez, Gloria Isabel. 2008. Modelo de cuantificación de riesgos laborales en la construcción: Ries-co. Tesis doctoral. Valencia. España. Universidad Politécnica de Valencia. 440p.

- Cortés, JM. 2007. Seguridad e higiene del trabajo: Técnicas en prevención de riesgos laborales. $9^{a}$ edición. Madrid. Editorial Tébar. 771p.

- Departamento de estadística. 2011. Reporte de accidentes laborales. Armenia. Hospital San Juan de Dios. (entrevista).

- Enshassi, Adnon. Choudry, Rafiq M. Abu Algumboz Moheeb. 2009. Seguridad en la construcción. Ingeniería de la construcción. Vol.24. ( $\left.\mathrm{N}^{\circ} 1\right)$ : 49-79p.

- Gabriel, Jesús E, Chavarri C, Francisco de Borja. 2010. Funciones y responsabilidades de los agentes que intervienen en seguridad en las obras de UE-15(1). Gestión practica de Riesgos laborales. ( $\left.{ }^{\circ} 67\right)$. $18 \mathrm{p}$.

- Gerencia sucursal del Quindío. 2012. Datos estadísticos de accidentes laborales. Compañía Positiva S.A. Armenia, Quindío.

- Gerencia. 2012. Obras de construcción de vivienda. Armenia. Mesa de la construcción y la infraestructura.

- Hoyos T, William. García S, Bertha. 2009. Estructura metodológica de gestión de calidad, medio ambiente y salud ocupacional. Universidad Eafit. Vol.45. ( $\left.\mathrm{N}^{\mathrm{o}} 155\right)$ : 65-66p.

- Howell, Gregory. 2004. Safety, quality and enviroment. 762-764p

- López A, Mónica. Martínez A, María Dolores. Martín G, Esther. 2011. Análisis de riesgos muscoesqueléticos. Ingeniería de la construcción. Vol. 26. ( $\left.\mathrm{N}^{\circ} 3\right)$ : 284-298.

- Macchia, JL. 2007. Prevención de accidentes en las obras: Conceptos y normativas sobre higiene y seguridad en la construcción. Buenos aires. Editorial Nobuko. 51p.

- Martínez B, Ciro. 2008. Estadística básica aplicada: Distribuciones muéstrales. $3^{\mathrm{a}}$ edición. Ecoediciones. 350p.

- Marooszely, M. Karim, Khalid. Davis, Steven. Nik, Artin. 2012. Lessons learn in developing effective performance measures for construction safety management.

- Ministerio de comercio, industria y turismo. 2012. Colombia: nuestros avances y retos para ser un destino de clase mundial. Agosto. Cartagena de indias.

- Mitropoulos, P. Howell, G. Reiser, P. 2009. Workers at the edge, hzard recognition and action.

- OIT (Oficina Internacional del trabajo). 1991. Manual de educción obrera: La prevención de los accidentes. México. 141p.

- OIT (Oficina Internacional del Trabajo). 2000. Seguridad y salud en el trabajo de construcción: El 
caso de Bolivia, Colombia, Ecuador y Perú. Documento de trabajo. Lima, Perú. 136p.

- Organización Internacional del Trabajo. 2005. Aseguramiento de riesgos laborales.OIT.

- Registros públicos. 2012. Base de datos de empresas sector de la construcción. Armenia. Cámara de comercio de Armenia.

- Sheafer, Richard L. Mindehall, William. Ott, Lyman. 2007. Elementos de muestreo. $6^{\mathrm{a}}$ ed. España. Paraninfo. 462p.

- Saurin A, Tarcisio. Formoso T, Carlos. Fabricio, B. 2004. A human error perspective of safety planning and control. 675-777p

- Saurin A, Tarcisio. Formoso T, Carlos. Guimaraes, BM. Soares, AC. 2002. Safety and production on integrated planning and control model.

- Saurin, T. Formoso CT. Cambria, FB. Howell, G. 2008. A cognitive systems engineering perspective of construction safety.

- Superintendencia de sociedades. 2008-2012.Desempeño del sector de infraestructura. Informe. Bogotá, D.C. Septiembre de 2013. 7p.

- Suratep. Empresa promotora de riesgos laborales. 2012. Una alianza hacía la seguridad con productividad y calidad para su empresa.

- Sauter., Steven L. JosephJ, Murphy. Lawrence R. Levi, Lemart. 1.998. Enciclopedia de salud en el trabajo. Chile. Chantal Dufresne, BA. Tomo II, 55p.

- UGT (Unión General de trabajadores). 2012. Informe de accidentes de trabajo. Enero - Diciembre 2011. Aragón, Madrid. 76p.

- Universidad de Cienfuegos. 2003. Procedimiento para la seguridad y la salud. Departamento de ingeniería industrial. Cuba. 9p.

- Universidad Libre. 2011. Revista colombiana de la salud ocupacional. Cali, 1-2p. (Publicación 2(3)).

- Vásquez M, Heliodoro. 1992. Productividad y seguridad en el trabajo. Problemática actual de la industria. $1^{\mathrm{a}}$ edición. México. Editorial Diana.

- Walsh, Kenneth. Souhney Anil. 2004. Agent-based modeling of worker safety behavior at the construction warface.

- Zaherawait, Zakarua. Zalija, H.H. 2008. Accidents at the construction site in northern area Malaysian experiencied. Vol.4. (No3). 106-116p. 